\title{
Consequences of injury and motivation to return to sports among athletes: a review literature
}

\author{
Mathew Jacob ${ }^{1}$, Joel Mathew Jose ${ }^{2 *}$
}

\section{ABSTRACT}

We all know that injury plays a big role in every athlete's life, but what happens after and the process concerned with coming back to sports is important. In this paper review, we try to connect injury and motivation among athletes and the demanding factors important for their return. With the help of scales such as RSSIQ, STAI XI, SIP 15, and SIRBS, we could come to conclusions about the correlations between injury and motivation. These scales also helped us find many important factors to consider, such as anxiety and what state the athlete sees them. Therefore, we can say that injury is dynamic, and the consequences should not be ignored. The people close and involved with the athlete should also be made aware of it and also their roles that could play a major part during recovery.

\section{Keywords: Motivation, Injury, Psychological effects after injury, Sports Rehabilitation}

$\mathrm{I}$

n today's world, where many people are desk-bound employees who drive to work and barely walk-in everyday life, daily physical activity has become more important than ever. Although daily exercise and physical activity are critical to preserving good health, both amateur and professional athletes are frequently injured. The scope of these injuries is wide. Athletes can sustain minor injuries that last only a few days. They can, however, be seriously injured and operatively treated. The recovery process will take a long time, leading to a massive amount of time for the athlete to be deprived of sports activities and training. These injuries can also lead to a sports career being ended. It is important to make the right diagnosis and to apply sufficient care to prevent permanent disabilities. When diagnosing sports injuries, the trauma process is very important. To gain knowledge about the trauma process, the athlete, or a witness to the wound should be asked.

Also, radiological procedures are instrumental. The radiologist must be told about the trauma process for an effective diagnosis. For successful treatment, early diagnosis is key. Missed cases appear to turn up later in the clinic with chronic issues. Injuries to sports require more than just acute trauma. Sports injuries are also microtraumas and overuse injuries and can lead to chronic pain and loss of functionality. In professional athletes, sports injuries are common; however, people who sport in gyms and parks or at home may also

${ }^{1}$ B.Sc. Clinical psychology student, AMITY University, Panvel, Mumbai, India

${ }^{2}$ M.Sc. Clinical psychology student, CHRIST (Deemed to be University), Hosur Road, Bangalore, Karnataka, India

*Responding Author

Received: January 06, 2021; Revision Received: February 10, 2021; Accepted: March 03, 2021

(C) 2021, Jacob M. \& Jose J.M.; licensee IJIP. This is an Open Access Research distributed under the terms of the Creative Commons Attribution License (www.creativecommons.org/licenses/by/2.0), which permits unrestricted use, distribution, and reproduction in any Medium, provided the original work is properly cited. 
suffer from these injuries. Any of the traumatic pathologies associated with such athletic activities are tendonitis, muscle sprains, and spasms, even meniscus or ligament ruptures. (Derin Cicek, 2020)

It is possible to describe a sports injury as an injury that happens during sports activities or exercise. This can be broadened to include injuries affecting sports and exercise participation and affecting athletes of all ages and all performance levels. The continuum from top professional to recreational athletes is reflected by patients seeking medical care at sports injury clinics. While the mechanism of an injury and its pathoanatomical association or diagnosis may be established, various athletes' effects can be quite different. There could be a loss of earnings and the possibility of losing your contract and even your future if you are a professional player. If you are a club manager, it could involve losing a valuable player and a replacement player's financial costs, maybe at a critical time. You would want to know how the injury will affect the player's current nutrition and physical exercise activities, whether the team doctor, physiotherapist, personal trainer, or coach. If you are the medic in charge, it will mean having to reassure the player and the other club employees that you have control of the situation. There are high stakes. They risk relapse or further injury if a player goes back too early, but they can request a second opinion if they are held back. An injury can result in significant family-related conflicts for younger athletes seeking to develop themselves in their sport. A young athlete unable to participate in their sport can be pressured by over-ambitious or over-protective parents and pressure from coaches and teammates. Injuries can mean losing daily physical and social activity and general health issues for recreational athletes, such as blood pressure, insulin regulation, or secondary problems from limping to the lower back. A squash shoulder injury can trigger a builder or plumber difficulties with their own business or raise questions about a police officer or firefighter's safety. Whoever offers care and guidance must stress the value of sport and exercise and the implications of an injury. They need to respect and understand but give advice based on facts. Advice must be carefully considered to inform a keen recreational tennis or golf player that they need to avoid playing because of an injury. During rehabilitation from even a very serious injury, there is often a range of choices for consideration. Total rest is rarely motivating and can be ill-advised due to the adverse impact that rest has on tensile tissue's strength and general health and the potentially fatal effects for some patients. An 80-year-old keen, daily golfer could die from the activity caused by the injury, suffering from a painful knee due to a meniscus tear. The knee could be operated on and repaired with arthroscopy within fifteen minutes, enabling him to play golf a week or two later; asking him to stop playing golf would be a shame and very wrong. The secret to success for doctors is to consult evidence-based guidelines for the understanding and diagnosis of an injury; to use accurate diagnostic methods, to take into account the patient's history and fitness level; and to be willing to confess to a lack of expertise and to refer the patient to someone who may know more. They must consider the changes and advances in sports medicine and the cultural variations in the treatment of these injuries that are taking place. Doctors should not consider that sports accidents are self-inflicted and advise their patients to stop doing foolish things. Osteoporosis and a general decrease in fitness due to inactivity, exercise, and sport are potent ways of keeping the population active and healthy in communities challenged by obesity. For example, 70 percent of keen runners may be affected by a lower limb injury during their career, typically by over-use; soccer players have a high risk of the traumatic ankle or knee injuries from tackles. Most sports injuries are unique to the sport and the level of participation. The frequency of soccer injuries ranges from 15 to 20 injuries per thousand hours of practice, with the highest risk during games. The figures are much higher for rugby: between 20 and 40 injuries per thousand hours of operation and a higher risk of injuries to 
the upper limb, especially those to the shoulder joint. Golf is a low-risk sport, but success and the ability to walk a five-kilometer course can be affected by a knee or shoulder injury. (Rolf, 2007)

\section{Classification of injuries}

Injuries to sports or results can be categorized according to either the cause of the injury or the type of affected body tissue. If injuries are categorized according to the cause, direct injury, indirect injury, and overuse injury are the three categories. If injuries are categorized according to affected body tissue, soft-tissue injury, and hard-tissue injury are the two types.

Direct injury: An external blow or force causes a direct injury. A collision with another individual can cause direct injuries. For example, a cricket ball or hockey stick is hit with an item during a rugby union tackle.

Indirect injury: In two ways, an indirect injury can happen:

The actual injury can take place some distance from the site of the impact. Falling on an outstretched hand, for example, may result in a dislocated shoulder. The injury is caused not by physical contact with an object or individual but by internal forces generated by the performer's actions, such as over-stretching, bad technique, exhaustion, and lack of fitness. Examples of these injuries are ligament sprains, hamstring strains, and tears.

Overuse injury: Overuse injuries occur when the bones and other connective tissues of the body are put under an unnecessary and repetitive force. In the early stages of these injuries, little to no discomfort could be encountered, and the athlete could continue to exert pressure on the injured site. This avoids giving the site the necessary time to recover. The harm gradually accumulates, and the affected site becomes inflamed and, ultimately, painful. When there is a shift in training habits, such as increasing training frequency or strength, overuse injury symptoms often arise, and the body cannot deal with the new pressures put on it. A significant number of injuries from overuse result from poorly structured training plans in which the athlete is not given enough time between rigorous sessions to heal. Usage of improper technique and poor equipment are other sources of overuse injuries. Athletes who use bad techniques or equipment to train and compete put extra stress on their bodies. Examples include elbow damage from the bad backhand technique or the use of a heavy tennis racquet and pain in the ankle or knee from an improper running style or from wearing inappropriate shoes.

Table of overuse injury

\begin{tabular}{|l|l|l|}
\hline Injury & Symptoms and signs & Possible causes \\
\hline \multirow{5}{*}{ Shin soreness } & Tenderness & Increased activity \\
& Pain in shins & Poor footwear \\
& Pain increases by running \\
and jumping & Postural imbalance \\
& Swelling & Muscle imbalance \\
\hline \multirow{5}{*}{ Knee pain } & Pain around knee & Increased activity \\
& The pain increased by sport, & Postural imbalance \\
& stairs, sitting, hills & Poor footwear \\
& Swelling & Muscle imbalance \\
& Discoloration & Growth spurt \\
\hline \multirow{3}{*}{ Heel pain } & Tenderness over heel Pain & Tight calf muscles \\
& increased by & Growth spurt \\
& running, jumping & Poor footwear \\
\hline
\end{tabular}

(c) The International Journal of Indian Psychology, ISSN 2348-5396 (e)| ISSN: 2349-3429 (p) | 174 


\begin{tabular}{|l|l|l|}
\hline Injury & Symptoms and signs & Possible causes \\
\hline \multirow{5}{*}{ Shoulder pain } & $\begin{array}{l}\text { Pain on certain } \\
\text { movements } \\
\text { Reduced movement } \\
\text { Local tenderness }\end{array}$ & $\begin{array}{l}\text { Increased activity, } \\
\text { e.g., swimming } \\
\text { Poor technique, e.g., swimming, } \\
\text { pitching, serving }\end{array}$ \\
\hline \multirow{5}{*}{ Elbow pain } & $\begin{array}{l}\text { Increased activity, e.g., golf, } \\
\text { Pain in and around the elbow } \\
\text { The pain increased by certain } \\
\text { activities, e.g., shaking, lifting, } \\
\text { gripping, Jarring }\end{array}$ & $\begin{array}{l}\text { Muscle imbalance } \\
\text { Poor technique } \\
\text { Change of grip } \\
\text { Lack of control }\end{array}$ \\
\hline
\end{tabular}

Source: International Journal of Informative and Futuristic Research [ IJIFR] Volume-1, Issue-3, (2013).

\section{Common sports injuries}

Sprains:_A sprain is when you stretch, twist, or tear one or more of your ligaments. Solid bands of tissue around joints are ligaments. They attach one bone to another, helping to keep the bones together and stable. In ligaments around joints in the ankle or knee, sprains frequently occur. There is no dislocation or splitting of the joint.

Sprain's symptoms include the following:

- Inflammation of pain (swelling)

- The Bruising

- In the affected area, limited movement.

Strains: A muscle strain is where strained or broken muscle tissues or fibers are found. Often a muscle strain is referred to as 'pulling a muscle.' Also, tendons may be stretched. The tough, narrow tissue at the end of a muscle that binds it to the bone is a tendon. A muscle that is overstretched or that over-contracts induce strains. Strains, particularly those involving running, jumping, or sudden direction changes, are prevalent in many sports. Including symptoms:

- Pain

- Spasm of the muscle

- A loss of strength in the muscle.

Dislocation: An injury in which the ends of your bones are pulled from their usual positions is a dislocation. Trauma resulting from a fall, a car accident, or a crash during touch or highspeed sports is typically the cause. Dislocation typically affects the larger joints of the body. In adults, the shoulder is the most common site of injury. It is the elbow in girls. If violently twisted the wrong way, your thumb and fingers are also vulnerable. The fracture will deform and immobilize your joint temporarily and lead to sudden and extreme pain and swelling.

Fractures: For individuals of all ages, fractures are a common injury. It may also be difficult to diagnose a fracture since it depends on the position and how the fragments are aligned. The difference is evident between a compound fracture and a simple fracture, and an X-ray can measure the form of the fracture. As their bodies are still developing, children can recover quicker than adults. A child's fracture might take just a few weeks to heal, while an older adult might take months to heal. Practicing protection and consciousness is the only way to prevent one from happening. 
Low back pain: Several problems with any part of the complex, interconnected network of spinal muscles, nerves, bones, discs, or tendons in the lumbar spine can cause lower back pain. Low back pain can be caused by disorders affecting the bone's lumbar spine, discs between the vertebrae, ligaments around the spine and discs, spinal cord and nerves, low back muscles, pelvic and abdominal internal organs, and skin covering the lumbar region. (ERDEMIR et al., n.d.)

\section{History of Sports injury and research}

The history of study on the psychosocial history of sports injuries dates back 35 years. In the more than three decades of research into athletic injury predictors, numerous papers have appeared in the literature, ranging from early psychodynamic injury formulations (Sanderson, 1977, 1978) to the generally more developed field-guiding models. There is little doubt about the importance of psychosocial factors on the risk of injury and the result of the injury. A reason for the limited recent study may be that there are procedural and logistic difficulties conducting such studies. The long-term goal of injury history research was to establish strategies to change the psychosocial risk factors found, such as reducing stress and enhancing coping, reducing the incidence of injury, and improving athletes' quality of life and sporting experiences. Intervention research is now the cutting edge of investigating sports injury and perhaps the most exciting aspect of this emerging sports science area. So far, minimal preventive research has been carried out, but what we have holds significant promise.

According to the Consumer Product Protection Commission, at least one week of nonparticipation is required for one-quarter of these injuries (Hardy \& Crace, 1990). Although several injuries are not serious or life-threatening, Burt and Overpeck (2001) estimated that 3.7 million children and adults visited emergency rooms for exercise and sports-related injuries for one year (1997 to 1998) in the United States. Gotsch, Annest, and Holmgreen (2002) estimated that the number of emergency room visits related to sport and recreation was 4.3 million from July 2000 to June 2001. These numbers illustrate the need for research to investigate the causes and prevention of sports injuries. Since the late 1990s, the European Home and Leisure Accident Monitoring System (EHLASS) has been collecting sports injury data in Europe from several countries. For example, in Austria's EHLASS study, it was reported that in 2000 there were 227,400 (29 per 1,000 person-years) sport-related injuries, with direct medical costs of EUR 66 million (USD 84 million). When figures for production losses are included, the costs amount to EUR 302 million (\$382 million in US dollars). In the Netherlands, around 1.5 million people are expected to suffer a sport-related injury per year, with more than 170,000 visiting emergency departments in hospitals. The reporting of EHLASS differs widely amongst European nations, and we could not find a consistent central finding for the results. (Williams \& Andersen, 2007)

\section{Psychological effects of the injury on athletes}

Many athletes practice in a constant state of pain or injury when meeting an elite level program's demand. The emotional distress endured by athletes with chronic injuries is not hypothesized to be inconsequential. (Shuer \& Dietrich, 1997)

There are an estimated seven million incidents associated with sports and recreation per year in the United States, not counting sports injuries that could go unreported, such as sprains and concussions. 521,578 individuals were treated for accidents related to bicycles, according to the National Sporting Goods Association. Additionally, 83 percent of novice or competitive runners suffer knee, ankle, and foot injuries that can temporarily or definitively

(c) The International Journal of Indian Psychology, ISSN 2348-5396 (e)| ISSN: 2349-3429 (p) | 176 
impair their quality of life. Sport and recreational accidents continue to escalate over time, despite attempts to minimize injuries with improvements in athletic equipment, coaching methods, and sports-specific conditioning. When athletes are injured, they undergo various emotions that are also more debilitating when they require a longer recovery. For example, a record-setting freshman of the year tailback and Heisman candidate for the University of South Carolina, Marcus Lattimore, sustained a series of sports injuries, including a torn Anterior Cruciate Ligament (ACL), dislocated kneecap, torn ligaments, and nerve damage. He was drafted by the San Francisco 49ers in the fourth round, through numerous surgeries and recovery. However, he decided to give up football after just a few days of practice due to discomfort and loss of faith in his knees' ability to work at the same level he previously had.

\section{Re-injury anxiety}

Reinjury anxiety is one of the most common psychological reactions faced by injured athletes and the most widely cited explanation provided by athletes for not returning to sports after ACL surgery. Reinjury anxiety or fear of rejuvenation, both used synonymously in sports injury literature, is described as irrational and crippling fear or anxiety that physical movements will result in painful rejuvenation. In a study of 49 recreation-level athletes who had undergone ACL surgery one year earlier, Trippet al. found that athletes with high reinjury anxiety showed lower confidence in their ability to return to sport. Several researchers have hypothesized that fear of injury is often present among athletes, particularly after a serious injury. Reinjury anxiety is associated with psychological changes, including reduced attention and self-confidence and increased distractibility and pain perception. Rejuvenation anxiety can also cause physiological changes, including over-excitation, which is noticeable by elevated heart rate, generalized muscle tension, and defense of the wounded site. As a result, an athlete who fears rejuvenation continues to establish a lack of confidence in the injured site, leading to hesitancy in results during recovery and returning to training and competition. Overall, both psychological and physiological responses to rejuvenation anxiety contribute to athletes falling into a pattern of inactivity that may lead to decreased body strength and flexibility and may result in athletes feeling greater pain. At the same time, they are involved, thereby increasing rejuvenation anxiety that perpetuates continued prevention.

\section{Psychological distress}

In addition to rejuvenating anxiety, athletes may experience depressive symptoms following injury. The severity of depressive symptoms may differ depending on the injury, mobility limitations, duration of recovery, and delay in returning an athlete to the sport or physical activity. Depressive symptoms may occur shortly after the injury. They may be associated with dissatisfaction due to immobility, difficulty in daily activities, and feelings of injustice and shock associated with the injury. Depressive symptoms can often have a delayed onset. They can be associated with a sense of social isolation, loss of abilities or resources, and a general lack of interest in training or competition that may lead to the loss of athletic identity. As a result, an athlete who needs surgery after a sports injury could be more vulnerable to depressive moods than an athlete who has less significant sports injuries due to a delay in returning to play. Besides, athletes reporting somatic symptoms before the injury can affect the duration of their recovery period. (Sheinbein, 2016)

In a retrospective study of Division, I football players, 33 percent of injured athletes recorded elevated levels of depressive symptoms using the Depression Scale Center for Epidemiological Studies compared to 27 percent of non-injured athletes. If an athlete is 
injured, there is a natural emotional response, including collecting medical injury details collected by the medical staff and the injury's emotional care.

\section{Emotional responses}

- Sadness

- Isolation

- Irritation

- Lack of motivation

- Anger

- Frustration

- Changes in appetite

- Sleep disturbance

- Disengagement

Athletic trainers, team doctors, and players, coaches, and administrators must recognize that emotional reactions to injury are common. Still, troublesome reactions either do not resolve, escalate over time, or where the severity of symptoms tends to be excessive.

\section{Persistent symptoms}

- Alterations of appetite

- Sleep disturbance

- Irritability

\section{Worsening symptoms}

- Alterations of appetite leading to disordered eating

- Sadness leading to depression

- Lack of motivation leading to apathy

- Disengagement leading to alienation

\section{Excessive symptoms}

- Pain behaviors

- Excessive anger or rage

- Frequent crying or emotional outbursts

- Substance abuse

The study examined whether gender and seriousness can affect the emotional response and coping strategies of injured athletes and the relationship between coping strategies and emotional responses. Forty-three male and female subjects from nine different sports offered at four midwest intercollegiate institutions were recruited for the research. Subjects were asked to complete all three inventories within two days of the accident, and then every week before they returned to service. There is also a disparity in the gender response to injuries. A one-way study of variation was used to report variations between gender and injury severity. Males reported feeling more surprised than females. Gender discrepancies emerged with coping methods, and females had a greater tendency than males to use emotion-focused coping. The findings revealed few differences between male and female emotional responses to injury. Females may be more likely than males to deal with emotionally focused injury techniques. (Putukian, 2016) (Eaton, 1996) 


\section{After injury - Rehabilitation and motivation to return}

Athletes' ability to contribute to recovery and their importance to the rehabilitation process affects their cognitive, emotional, and behavioral responses to the rehabilitation of injuries. Thus, the way athletes view their injury rather than the fact that the injury happened has a vital role in recognizing athletes' emotional reactions, such as depression, rejuvenation, and sorrow.

Johnston and Carroll found that athletes who showed a strong fear of rejuvenation often had some behavioral reactions, including, but not limited to, being reluctant, not making $100 \%$ effort, and being wary of injury-provoking scenarios. They also found out that the athletes who were positively measured in their injury recovery recorded a feeling of pleasure and relaxation that promoted improved rehabilitation commitment. On the other hand, athletes who negatively judged their injury recovery reported feeling depressed, which led to hesitancy and caution in completing their rehabilitation program exercises. Injured athletes may find that the emotional and informational support given by athletic trainers, doctors, or specialists familiar with the recovery process is more effective in managing stress associated with their injury than what is offered by family and significant others. (Sheinbein, 2016)

Returning to sport following a serious injury can be a daunting task for certain athletes. Both anecdotal accounts and empirical investigations suggest that returning athletes can encounter fears associated with worries regarding their ability to perform up to pre-injury standards and pressures to meet clear return deadlines. It has also been suggested that athletes who resume competing after a serious injury can experience heightened competitive anxiety, concentrate needlessly on the injured region, and struggle to recover their technical skills and abilities. Eventually, such negative thoughts and experiences may increase the likelihood of rejuvenation and may have a detrimental impact on athletes' morale and postinjury results. (Podlog \& Eklund, 2005).

\section{Psychological intervention}

The defined role of psychiatry in injury research is not a new idea. Ten years ago, Tremblay and Peterson concluded that accident prevention initiatives lacked the psychology community's requisite support. That injury deserved to be a higher priority on our public health agenda. Psychologists and other behavioral psychologists have a considerable experience that can and can be applied to injury prevention efforts. While psychologists currently contribute a wide range of skills to injury research across various injury-related fields, including crime, transportation, and recovery, and have done so for decades, their numbers in the field are relatively small. To fill the void between behavioral scientists in injury, we agree that it is vitally necessary for psychologists currently researching injury to inspire those in the field to consider how their work can be applied to injury research. (Stavrinos \& Schwebel, 2009)

The research was undertaken by Laura.M, Schwab Reese, Ryan Pittsinger, and Jingzhen Yang, which included studies evaluating the efficacy of treatments to reduce post-injury psychological effects. Psychological interventions were described as those that used psychological methods, including visualization, goal-setting, relaxation, and other common techniques applied during the post-injury recovery era.

They included intervention experiments with target groups of seriously injured competitive and recreational athletes aged 17 years and older. Serious injury is described as injurycausing at least three weeks away from the play. 
Their studies have shown that therapeutic strategies using directed visualization, goal setting, or relaxation are often correlated with reduced negative psychological effects, increased coping, and reduced anxiety re-injury. Specifically, guided imagery/relaxation is associated with better psychological functioning and decreased anxiety re-injury. However, the setting of the target was not specifically correlated with the reduction of negative psychological effects. Other therapeutic strategies, such as micro-counseling skills, ACT, and written disclosure included in this study, have shown reduced negative psychological effects, enhanced psychological coping, and reduce anxiety due to re-injury. (Reese et al., 2012)

An integrated model of psychological response to the sports injury and rehabilitation process.

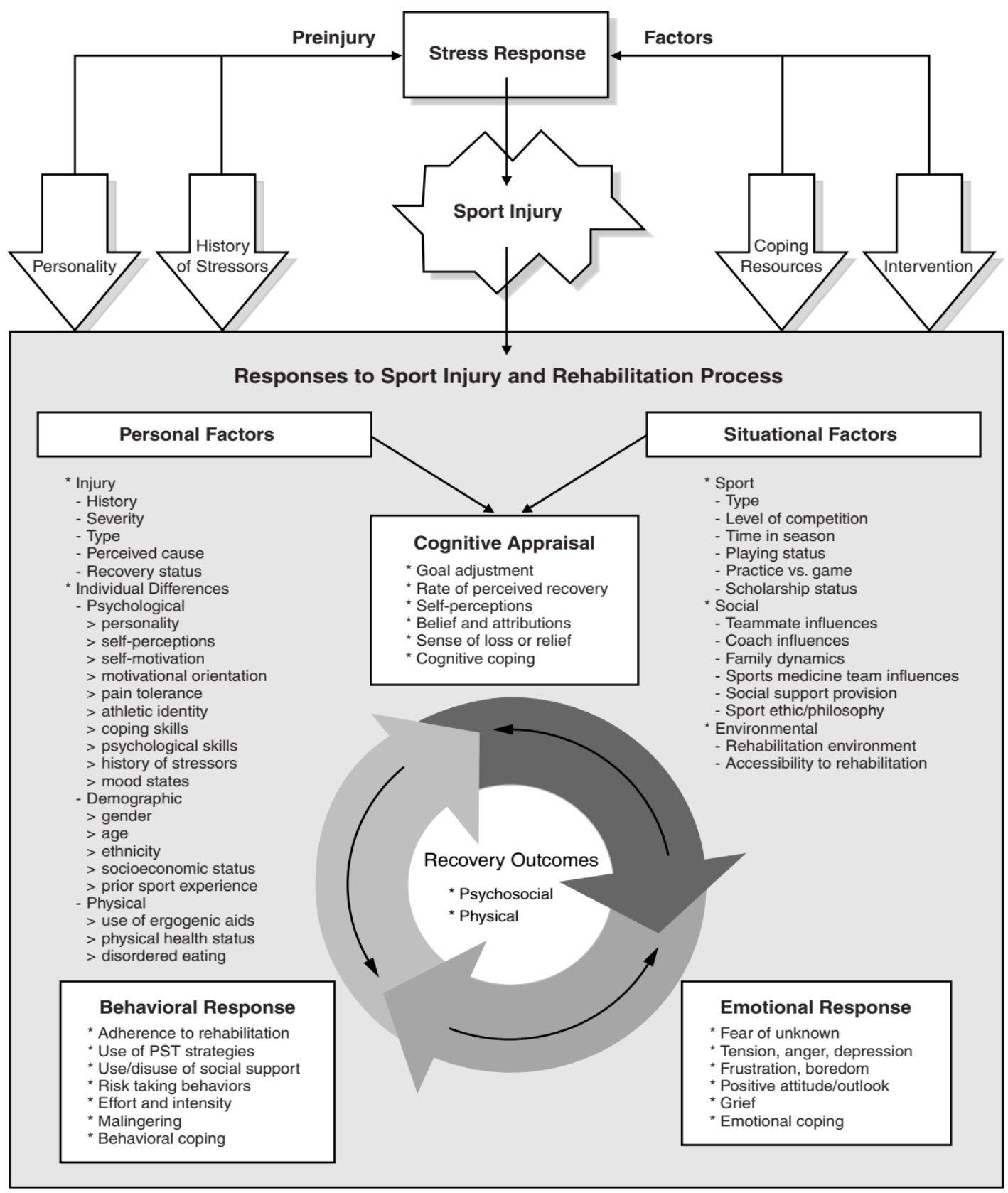




\section{Future of injury research}

Future studies need to analyze several predictors and moderator variables and then evaluate the varying patterns through which they interact to influence the hypothesized stress response and injury vulnerability and resilience. One challenge in researching several predictors and moderator variables and, accordingly, subgroups of individuals with extreme variables need very large sample sizes. The practical need suggests that potential researchers should consider including researchers from a variety of geographical areas. The additional advantage of such an approach is the improved generalizability of the findings. The probability of various types of injuries and the timing of injuries should also be investigated in the light of the preceding suggestions. Researchers should continue to use prospective prototypes and collect reliable injury data to execute these and other recommendations. (Williams \& Andersen, 2007)

\section{METHODOLOGY}

\section{Sample}

According to two main studies in reference (Leslie Podlog and Robert C. Eklund, Return to Sport After Serious Injury: A Retrospective Examination of Motivation and Psychological Outcomes) and (Robert Masten, Psychological factors of rehabilitation of athletes after a knee injury). The samples taken were roughly 480 participants of elite athletes and school division professional players. Out of which 240 were male, and 240 were female.

\section{Instruments}

Four measures were used in this study,

1. The Return to Sport After Serious Injury Questionnaire (RSSIQ): The Return to Sport After Serious Injury Questionnaire was developed to assess an injured athlete's perceived psychological outcomes of returning to sports. It is a 21 item questionnaire derived from examining the psychosocial sport-injury literature and the first author's personal experience as a college athlete who had experienced numerous surgical procedures and lay-offs resulting from the injury. These items assessed cognitive, affective, and behavioral aspects of the athlete's post-injury return outcomes.

2. State-Trait Anxiety Inventory (STAI X1): STAI X1 is one of the most widely used anxiety scales. The scale measures anxiety as a state. The state of anxiety is characterized by a temporary increase in excitement, while moderate anxiety is considered a normal stress reaction. The inventory consists of 20 items, and participants respond to items on a 4 point rating scale.

3. Sports Inventory for Coping With Pain (SIP 15): The Sports Inventory for coping with Pain (SIP-15) is a 15 item questionnaire measuring an athlete's psychological response or coping with pain. The questionnaire subscales measure the following three dimensions: Coping through Direct Action, Catastrophizing, and Somatic Awareness. It has a 5 point rating scale to which the participants have to answer. SIP 15 is a reliable and valid instrument for measuring coping with pain that athletes need to employ when recovering after an injury.

4. Sports Injury Rehabilitation Beliefs Scale (SIRBS): Sports Injury Rehabilitation Beliefs Scale (SIRBS) measures the factors proposed by the Protection Motivation Theory (PMT), a theory on how individuals protect themselves. The SIRBS scale consists of 19 items and assesses injured athlete's rehabilitation behaviors. Participants respond to all items on a 7 point rating scale. 


\section{Procedure}

According to both the tests referred to here; first, all the participants were made aware of the tests and their purposes. All athletes were provided with information sheets regarding the study's purposes, and their informed consent was obtained. After which, all the athletes completed the questionnaire given to them with its respective purposes. The respective code of ethics was followed according to the psychologist association concerned during the entire research procedure.

\section{RESULTS}

Principal-axis factor analyses with direct oblim rotation were conducted to reduce RSSIQ item responses into a smaller number of interpretable factors for subsequent analyses. The first analysis revealed five factors with values exceeding 1.0 after the direct oblim rotation. A series of principal-axis analyses with direct oblim rotations was subsequently conducted to determine the items to be used for a 2-factor solution. A total of 15 items were tested in the final solution. The final solution revealed that the featuring items had a strong association with two factors. This solution accounted for approximately $56 \%$ of the variability among these RSSIQ items. To establish a relationship between psychosocial variables as well factor analysis was used to enable weighting of variables. The result was that the factor in question was loaded negatively with anxiety as a state. We were also able to predict the success of an athlete's psychological rehabilitation based on the changes in the measured psychosocial variables through rehabilitation.

\section{DISCUSSION}

In conclusion, injury is dynamic and yet to be learned side by side for psychologists. The causes of injuries, although the consequences are known, are still to be fully understood, particularly the psychosocial variables involved. It can be safely assumed that a lot remains to be known and a lot that can be achieved with the present knowledge to minimize psychosocial effects to a minimum.

Sports psychologists should begin educating coaches and athletic trainers on psychosocial factors that can impact injury. Such information could lead coaches to increase their understanding of non-sport aspects of an athlete's life that could cause stress. Increased sensitivity can also contribute to greater social support, possibly buffering some of the negative effects of stress. Coaches and sports psychologists should also consider developing rehabilitation services for athletes with high-risk injury profiles.

According to the authors, this research aimed to find the association between motivation and returning to sports from a serious injury and its psychological outcomes. Empirical data, according to the study, strongly poses a positive association between motivation and beneficial consequences. (Williams \& Andersen, 2007).

\section{REFERENCES}

Derin Cicek, E. (2020). Sports Injuries.

Eaton, D. S. (1996). A study of the emotional responses and coping strategies of male and female athletes with moderate and severe injuries.

Podlog, L., \& Eklund, R. C. (2005). Return to sport after serious injury: A retrospective examination of motivation and psychological outcomes. Journal of Sport Rehabilitation, 14(1), 20-34. 
Putukian, M. (2016). The psychological response to injury in student athletes: A narrative review with a focus on mental health. British Journal of Sports Medicine, 50(3), $145-148$.

Reese, L. M. S., Pittsinger, R., \& Yang, J. (2012). Effectiveness of psychological intervention following sport injury. Journal of Sport and Health Science, 1(2), 7179.

Rolf, C. (2007). The sports injuries handbook: Diagnosis and management. A\&C Black.

Sheinbein, S. (2016). Psychological effect of injury on the athlete: A recommendation for psychological intervention. AMAA Journal, 29(3), 8-11.

Shuer, M. L., \& Dietrich, M. S. (1997). Psychological effects of chronic injury in elite athletes. Western Journal of Medicine, 166(2), 104.

Stavrinos, D., \& Schwebel, D. C. (2009). The role of psychology in injury prevention efforts. Injury Prevention, 15(1), 69-69.

Williams, J. M., \& Andersen, M. B. (2007). Psychosocial antecedents of sport injury and interventions for risk reduction.

\section{Acknowledgement}

The author appreciates all those who helped in the study and helped to facilitate the research process. The authors are indebted to their families and friends for their constant support, reviews, and comments.

\section{Conflict of Interest}

The author declared no conflict of interest.

How to cite this article: Jacob M. \& Jose J.M. (2021). Consequences of injury and motivation to return to sports among athletes: a review literature. International Journal of Indian Psychology, 9(1), 172-183. DIP:18.01.020/20210901, DOI:10.25215/0901.020 